\title{
Energy Management Scheme in Hierarchical Integrated Energy Systems with Coalition
}

\author{
Nayana Shetty \\ Associate Professor, \\ Department of Electrical and Electronics Engineering, \\ NMAMIT, Nitte, \\ Udupi, Karnataka, India \\ nayanar401@gmail.com
}

\begin{abstract}
Often, coalitions are formed by the hierarchical integrated energy systems (HIESs) and their evolutionary process which is driven by the benefits of stakeholders and consolidate energy consumers and producers. Several literature have failed to analyze the operation of HIES under the impact of multiple coalitions. At the lower level, multiple users, in the middle level, the multiple distributed energy stations (DESs) and at the upper level, one natural gas and one electricity utility company structure is used for analyzing the HIES operation with a trading scheme. The Lagrange function is used for deriving the optimal operation strategy based analytical function for each probable coalition and each market participant comprising of users and the DESs. It is evident from the results that in a single coalition, the profits linked to other DESs will decrease while increasing the profit of one DES with technological enhancements, users show an aversion towards DESs with high generation coefficient while they are attracted to the ones that enable reduction of heat and electricity price. Maintaining their isolation is preferred by high heat and electricity consuming DESs at the same energy price. Other coalitions and their operations are not affected by the change in parameters of one coalition.
\end{abstract}

Keywords: Distributed energy station, Hierarchical integrated energy systems, Smart metering, Demand Response, Energy Management

\section{Introduction}

The conventional structures of energy supply and human social development has encountered several contradictions due to the current environmental crises [1]. There is a global increase in 
the need for energy supply and its structural transformation. For multi-energy production, the consumer demands are satisfied by rapid global development of the distributed energy stations (DESs) that are situated at the consumer sites [2]. The mismatch between energy requirement and production is mitigated by improving the demand response potential by increasing the energy system reliability and flexibility through DESs as compared to the traditional municipal energy systems. The power distribution related businesses were paid significant attention during the second energy market reform implementation at China in 2014 [3]. Businesses were provided with social capital due to these approvals. Determination of energy price mechanism by the market is performed gradually by realizing the energy trade marketization and breaking the monopoly in the energy market, which is the energy market reforms ultimate aim. The hierarchical integrated energy systems (HIESs) are formed gradually by encouraging the entrance of more DESs into the market. There is a large improvement of the energy market flexibility as information technology is developing rapidly [4].

More choice is provided to the consumers and energy producers with the increase in data exchange between the stakeholders [5]. The one-to-one relationship between the consumers and producers has evolved to a great extent over the past. The market equilibrium prices are used for supplying energy to multiple consumers via multiple energy producers simultaneously [6]. The coalition mentioned in this study refers to such groups consisting of multiple consumers and producers. When compared to the conventional energy systems, the complexity and flexibility of energy market mechanism is high due to the incorporation of integrated energy market with multiple coalitions of different scales [7]. The study also focuses on understanding the various market decisions made by stakeholders within and outside the coalition, the coalitions formed by specific consumers and producers and integrated energy market with respect to the conditions for appearance of coalitions.

\section{Literature Survey}

It is essential to have a hierarchical formation in the continuously varying energy market for examination of the responses of several participants [8]. The electricity market is targeted so far for examination of energy trading as well as scheduling within hierarchical energy systems in existing literature. Game theory, multilevel optimization and multiagent 
Journal of Electrical Engineering and Automation (EEA) (2021)

Vol.03/ No.01

Pages: $34-43$

https://www.irojournals.com/iroeea

DOI: https://doi.org/10.36548/jeea.2021.1.004

approaches are applied on a wide scale for analysis of interaction of energy among multiple users in smart grid environment [9]. In distributed microgrids, optimal management is performed by examining multi-agent-based coordination and performing microgrid optimization by reviewing the multi-agent system reports by means of multi-agent approaches [10]. In case of standalone microgrid, the associated energy management is performed by means of a cooperative multi-agent system with the help of a distributed reinforcement learning scheme. An integrated building and microgrid system is controlled in an optimal manner while monitoring the energy management though an multi-agent-based ontology-driven system. Bilevel optimization and other multi-level optimization schemes are used for maximizing profits for marketers and energy generation industries by identifying optimal decisions [11].

In lower level, the demand-response aggregators and at the upper level, the transactive price signal between the electrical utility companies are calculated by the bi-level optimization model [12]. Karush-Kuhn-Tucker (KKT) conditions are used for transforming the model into a mixed-integer quadratically constrained program [13]. The bi-level optimization problem, a developed hierarchical decision-making framework model is also transformed into a linear single-level problem using KKT conditions. In a microgrid based on combined heat and power, the decision-making issues may be addressed using a multi-follower bi-level programming scheme. For electricity trading, the demand-response model may be described using Stackelberg game approach [14]. From a specific location, the real site building information is validated by developing interaction strategies between buildings and grids using Stackelberg game technique. An integrated technique is used for solving the mixed integer non-linear program formulated by the objective function of the consumer for managing the power grid demand site using an event-triggered game-theoretic approach [15]. The peer-to-peer energy trading is examined by several studies with the fast advancements in blockchain technology. Blockchain and smart contract is used for improving the reliability of the system by aligning the demand and supply using the demand side management schemes. Blockchain technology is used in power distribution systems for optimizing the physical and financial operation through analysis of the networked microgrids based technique. 


\section{Proposed Work}

A three-level hierarchical structure based HIES is considered for in this paper. One electricity and one gas utility company is considered in the higher level of the HIES. The multiple DES based middle level is provided with gas and high-voltage electricity by the two utility companies. The end users and provided with heat and low-voltage electricity obtained by the utility companies derived from the DES based transformed gas and high-voltage electricity. Several private enterprises also exist other than the utility companies that own most of the DESs. In future, the probability of private enterprise dominating the DES market is high based on the reform plans in the energy market. The original energy producer-consumer pair is formed when the user's energy demands are met by one DES. The user may be located at various district levels, neighborhood and buildings. For economic reasons, coalitions may be formed by consolidating various energy producer-consumer pairs with the advancement and evolution of HIES.

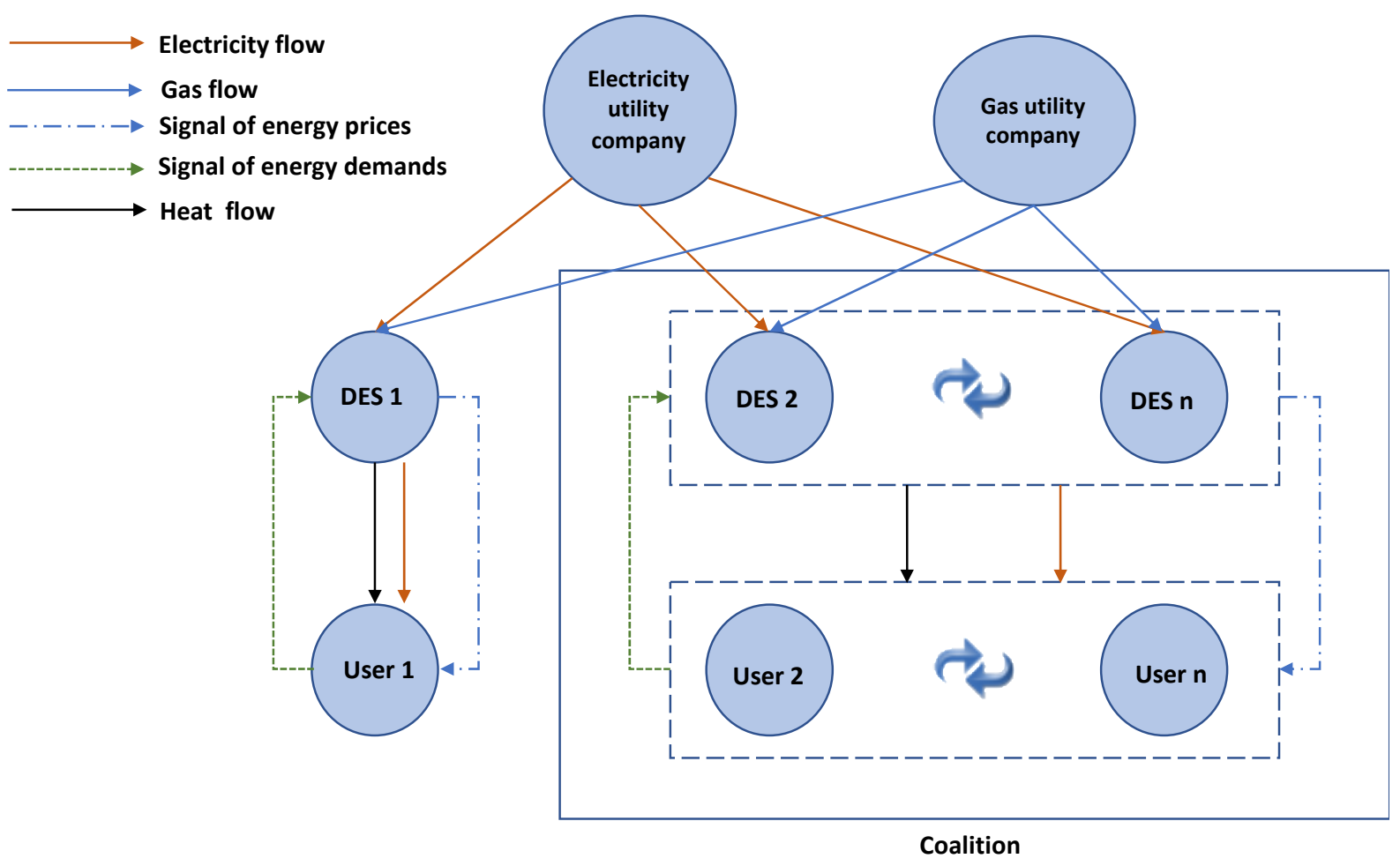

Fig. 1. DES integrated HIES framework with coalition 
Based on variation in energy prices, switching between energy providers is made possible for the energy users while breaking the barriers between participants using coalitions. In HIES, two scenarios are considered with respect to energy trading- with and without coalitions. When coalition does not exist, the energy and information transaction among multiple energy producer-consumer pairs stops from occurring. Among several coalitions, the data interaction and free energy causes middle level satisfaction of multiple DESs for lower level users energy demands. The probability of considering a coalition based special case scenario as one without coalition is high. Figure 1 represents the scenario with coalition. During smart metering, among several participants, multi-directional communication is established. This helps in attaining optimized HIES operation that enables demand information and price exchange while establishing smart metering.

\section{Results and Discussion}

The users, stakeholders, DES and utility company parameter values are analyzed. The energy trading results and the impact of system parameters may be illustrated by performing sensitivity analysis. MWh unit is used for measuring heat and electricity. $\$ / M W h$ is the measure for the cost of energy per unit. The operation strategy of each participant is derived by means of an optimal analytical solution. When there is a large number of providers and large population regime, the modeling and analysis is performed. Individual actions does not largely affect the market price in such a scenario. The properties considered for a perfect competitive energy market include equal or lesser market equilibrium price and marginal supply cost as called by the producers, consumers entering the market by voluntary payment of the market equilibrium price and overall voluntary participation. 
DOI: https://doi.org/10.36548/jeea.2021.1.004

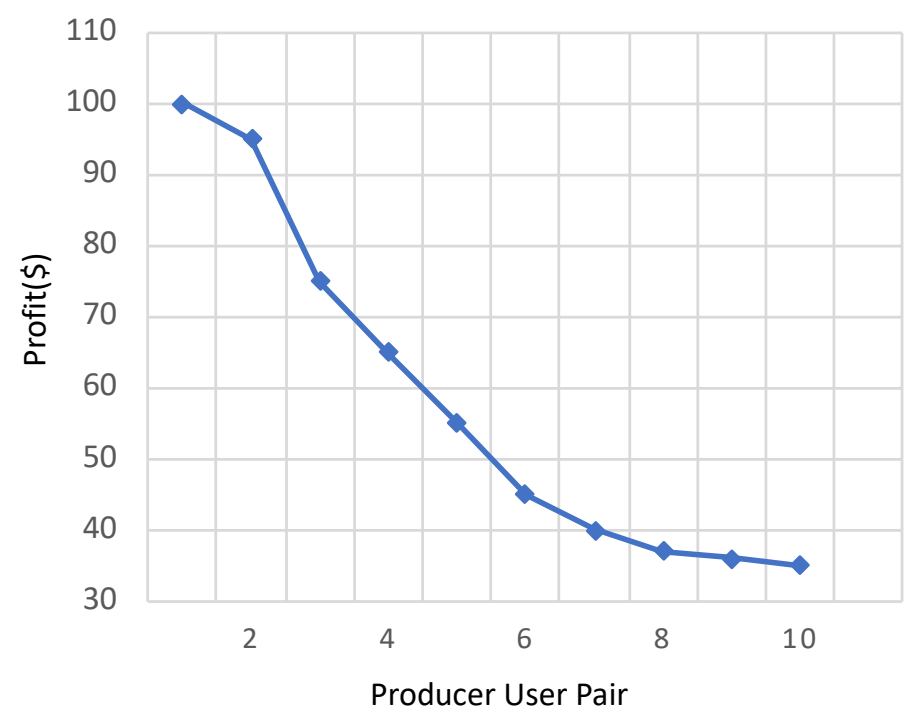

Fig. 2. DES coalition impact on stakeholder profit with increase in producer-user pairs

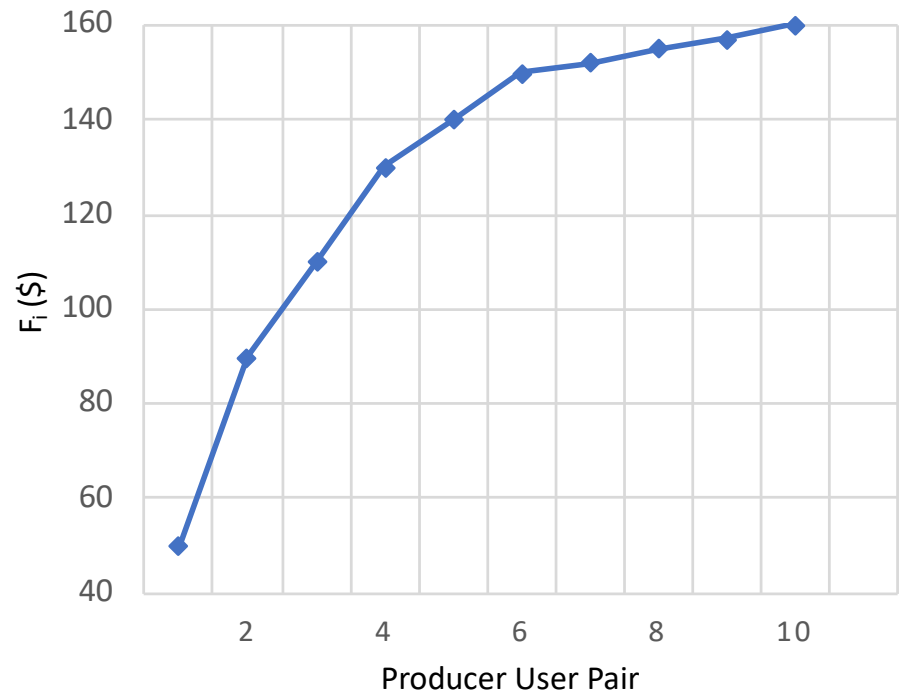

Fig. 3. User coalition impact on stakeholder profit with increase in producer-user pairs

During coalition, the profit of each DES based on the number of producer-user pairs is estimated. Figure 2 and 3 indicates that maximum crowding out of other DESs is performed by each DES. The profit of each DES decreases as the total producer-user pair count increases. There is a decrease in the market energy price due to the competition as the total producer-user pair count increases. From the users viewpoint, more producer-user pairs are accommodated. As the total producer-user pair count increases, the welfare of the users increase with the increase in energy demand. Figure 4 and 5 indicates multiple cases with varying energy prices 
and different stakeholders. There is a fluctuation in the parameters of various stakeholders within a certain range. The energy market interaction and energy trading is impacted by this fluctuation.

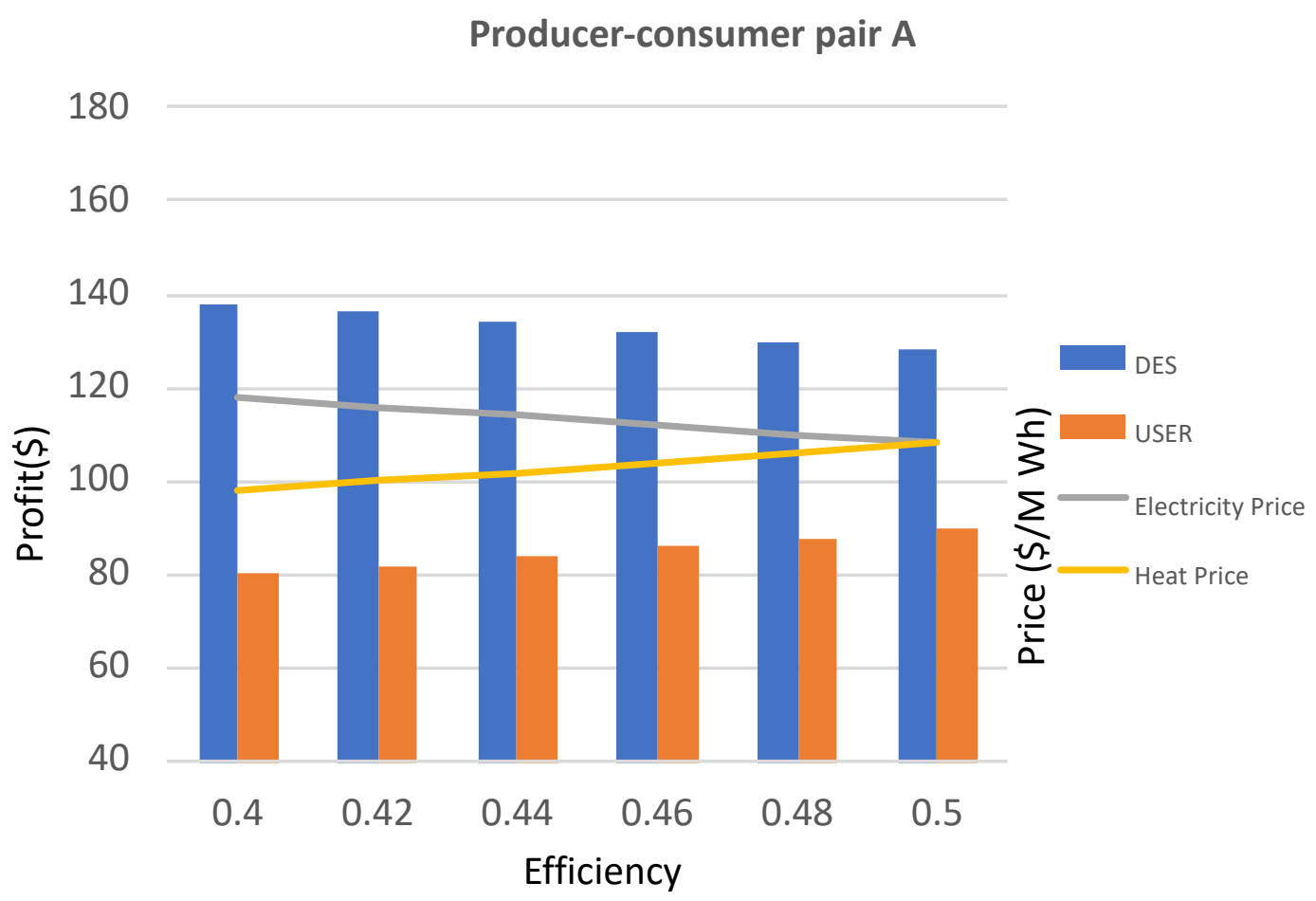

Fig. 4. Comparison of efficiency with respect to the cost and profit

The impact of system efficiency, generation coefficient and user parameters are estimated. There is no impact of one coalition on another in HIES with multiple coalitions. The operation of stakeholders in other coalitions are not impacted by the change in parameters in one coalition for this reason. Within a coalition, the profit of all DESs may be increased by the coalition formation. Isolation and securing the vested interest of original customers who consume more energy at the same cost in the DES and low generation coefficient based DES is to be maintained. Formation of large coalitions are preferred by the users as the energy prices may be reduced with the increase in market competition. The users change suppliers when unreasonably high prices are quotes by DESs in order to cooperative with other DESs leading to a decline in profit. 


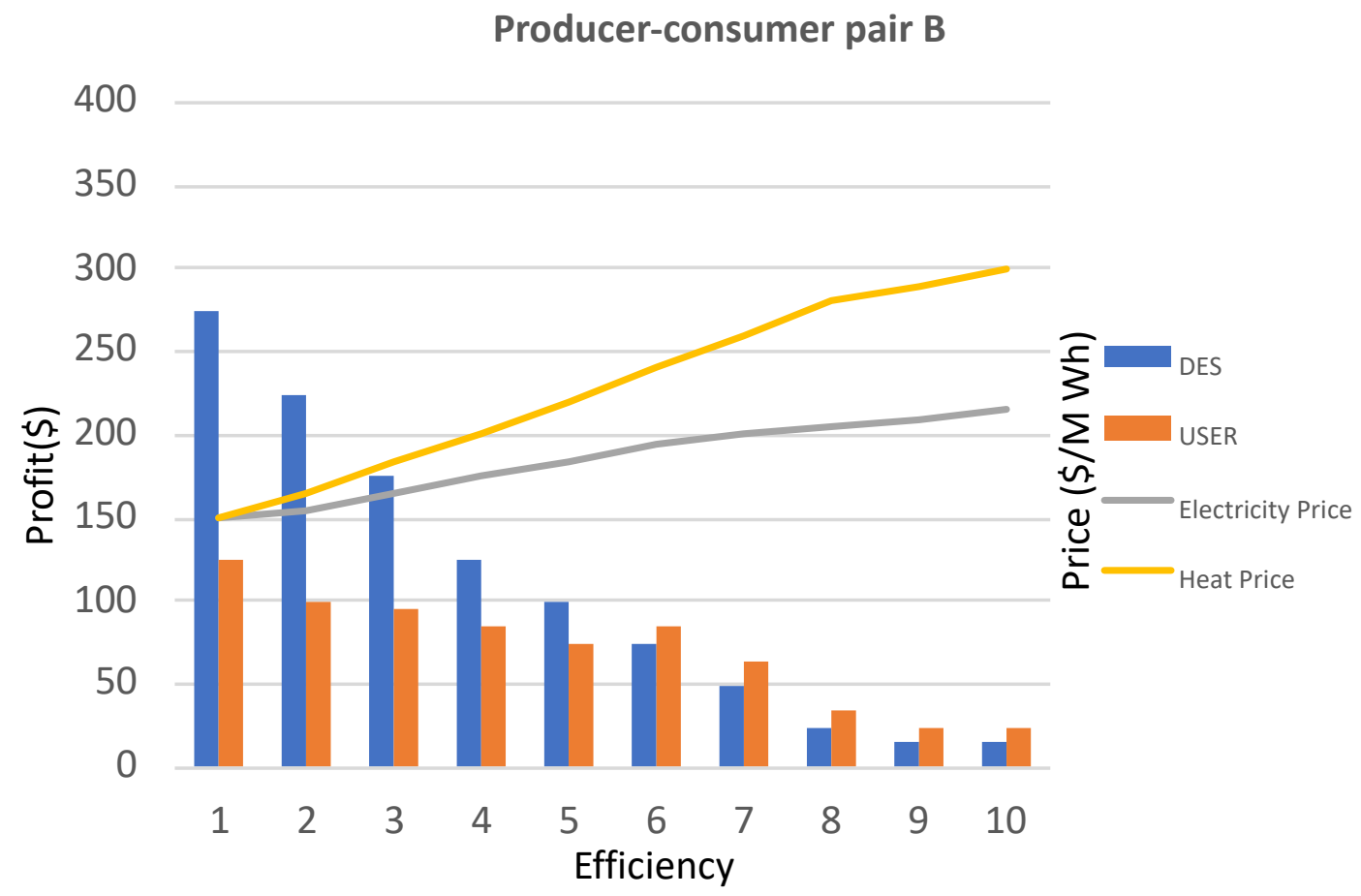

Fig. 5. Comparison of DES generation coefficient with respect to the cost and profit

In multiple coalition based three-level HIES, the interactions and energy trading mechanisms are analyzed and established. Each market participant and their optimal operation strategy and its analytical solution is derived using Lagrange's function. With the formation of coalitions, the impact of stakeholders on relative parameters are estimated and represented graphically. In most cases, a win-win strategy is established with the coalition formation. Optimized HIES operation is achieved by enabling demand and exchange price information through smart meters while establishing multi-directional communication between various participants.

\section{Conclusion}

The system operation is significantly affected by the multiple-coalitions that occur during the development of HIES. In a three level HIES, optimal strategies are obtained from various stakeholders to derive the analytical from of equilibrium energy prices. Three energy producer-consumer pairs are available in the HIES initially. Investigation of several HIES coalitions with respect to the impacts of participant parameters and structures as well as energy 
Journal of Electrical Engineering and Automation (EEA) (2021)

Vol.03/ No.01

Pages: $34-43$

https://www.irojournals.com/iroeea

DOI: https://doi.org/10.36548/jeea.2021.1.004

interaction among users, DESs and utility companies is performed. Real market users, DESs and governments may be benefited for decision making using the practical guidance and theoretical support obtained by the results. In order to simplify the analysis, same number of energy consumers and producers are assumed in the coalition. Future investigations may involve varying or increasing this number. It is also possible to perform examination of cost allocation and formation process in coalitions using game theory technique.

\section{References}

[1] Gholinejad, H. R., Loni, A., Adabi, J., \& Marzband, M. (2020). A hierarchical energy management system for multiple home energy hubs in neighborhood grids. Journal of Building Engineering, 28, 101028.

[2] Bahmani, R., Karimi, H., \& Jadid, S. (2021). Cooperative energy management of multienergy hub systems considering demand response programs and ice storage. International Journal of Electrical Power \& Energy Systems, 130, 106904.

[3] Coelho, V. N., Cohen, M. W., Coelho, I. M., Liu, N., \& Guimaraes, F. G. (2017). Multiagent systems applied for energy systems integration: State-of-the-art applications and trends in microgrids. Applied energy, 187, 820-832.

[4] Fan, S., Ai, Q., \& Piao, L. (2018). Hierarchical energy management of microgrids including storage and demand response. Energies, 11(5), 1111.

[5] Worku, M. Y., Hassan, M. A., \& Abido, M. A. (2019). Real time energy management and control of renewable energy based microgrid in grid connected and island modes. Energies, 12(2), 276.

[6] Li, L., \& Yu, S. (2020). Optimal management of multi-stakeholder distributed energy systems in low-carbon communities considering demand response resources and carbon tax. Sustainable Cities and Society, 61, 102230.

[7] Lahon, R., \& Gupta, C. P. (2018). Energy management of cooperative microgrids with high-penetration renewables. IET renewable power generation, 12(6), 680-690.

[8] Jing, R., Xie, M. N., Wang, F. X., \& Chen, L. X. (2020). Fair P2P energy trading between residential and commercial multi-energy systems enabling integrated demand-side management. Applied Energy, 262, 114551. 
[9] Prinsloo, G., Dobson, R., \& Mammoli, A. (2018). Synthesis of an intelligent rural village microgrid control strategy based on smartgrid multi-agent modelling and transactive energy management principles. Energy, 147, 263-278.

[10] Saifuddin, M. R. B. M., Logenthiran, T., Naayagi, R. T., \& Woo, W. L. (2019). A nanobiased energy management using reinforced learning multi-agent on layered coalition model: Consumer sovereignty. IEEE Access, 7, 52542-52564.

[11] Wolsink, M. (2020). Distributed energy systems as common goods: Socio-political acceptance of renewables in intelligent microgrids. Renewable and Sustainable Energy Reviews, 127, 109841.

[12] Bayram, I. S., \& Ustun, T. S. (2017). A survey on behind the meter energy management systems in smart grid. Renewable and Sustainable Energy Reviews, 72, 1208-1232.

[13] Wang, H. Flexibility Management in Renewable Energy Source Operated Power Systems using Decision Support System.

[14] Bindhu, V. (2019). Green cloud computing solution for operational cost efficiency and environmental impact reduction. Journal of ISMAC, 1(02), 120-128.

[15] Vijayakumar, T., \& Vinothkanna, M. R. (2020). Efficient Energy Load Distribution Model using Modified Particle Swarm Optimization Algorithm. Journal of Artificial Intelligence, 2(04), 226-231. 